\title{
High Mallampati score and nasal obstruction are associated risk factors for obstructive sleep apnoea
}

\author{
G. Liistro*, Ph. Rombaux*, C. Belge*, M. Dury ", G. Aubert", D.O. Rodenstein*
}

\begin{abstract}
High Mallampati score and nasal obstruction are associated risk factors for obstructive sleep apnoea. G. Liistro, Ph. Rombaux, C. Belge, M. Dury, G. Aubert, D.O. Rodenstein. (C)ERS Journals Ltd 2003.

ABSTRACT: Induced nasal obstruction can cause obstructive apnoeas in healthy subjects during sleep, but the relationship between nasal resistance measured during wakefulness and obstructive sleep apnoea syndrome (OSAS) is weak. It was postulated that if the subjects could not breathe through the nose, the oral airway must be used, but if this airway is narrowed as well, then it could precipitate sleep-disordered breathing (SDB).

Nasal patency, Mallampati score (MS), neck circumference and body mass index were measured in 202 subjects referred to the authors' hospital to undergo a full-night polysomnography for suspicion of SDB.

A significant correlation was found between the MS and apnoea/hypopnoea index measured during sleep. However, the relationship between these parameters was only significant in patients with nasal obstruction. The relative risk of having OSAS with a MS of III or IV was 1.95 for the whole group and 2.45 in patients with nasal obstruction.

In conclusion, a high Mallampati score represents a predisposing factor for obstructive sleep apnoea syndrome, especially if it is associated with nasal obstruction. These patients merit special attention from both the sleep physician and the anaesthetist.

Eur Respir J 2003; 21: 248-252.
\end{abstract}

\author{
*Pneumology, ${ }^{\#}$ Ear, Nose and Throat, \\ and "Neurophysiology units, Cliniques \\ Universitaires St-Luc, Université \\ Catholique de Louvain, Brussels, \\ Belgium. \\ Correspondence: G. Liistro \\ Service de Pneumologie \\ Cliniques Universitaires St-Luc \\ 10 Av Hippocrate \\ 1200 Brussels \\ Belgium. \\ Fax: 3227642831 \\ E-mail: Giuseppe.liistro@clin.ucl.ac.be \\ Keywords: Aetiology \\ apnoea \\ sleep \\ syndromes
}

Received: November 62001

Accepted after revision: July 52002
The obstructive sleep apnoea syndrome (OSAS) is characterised by repetitive upper airway occlusions leading to hypoventilation, apnoea and sleep fragmentation. The clinical presentation of OSAS is rarely specific. Indeed, male sex, obesity and daytime somnolence are extremely frequent in the general population. The association between overweight, large neck circumference and OSAS is well known [1, 2]. Complex models integrating the major and minor complaints of the patients and physical examination have been proposed and are still under evaluation [3, 4].

One of the clinical tests most widely used by the anaesthetists during preoperative physical examination is the Mallampati score (MS) [5]. The goal is to assess whether the upper airways may be seen easily during tracheal intubation. The higher the score, the higher the risk of a difficult intubation. The link with a physical examination of OSAS patients is simple, as a high MS could be associated with a higher probability of sleep-disordered breathing (SDB), because the oral airway is smaller.

Another group has recently tested this hypothesis [4]. These authors associated miscellaneous elements of physical examination with a modified MS, but this approach remains difficult.

Nasal obstruction during sleep has been associated with an increased frequency of obstructive apnoeas and hypopnoeas [6-10]. If a given subject has partial or total nasal obstruction, they must breathe through the mouth. But if the oral airway is also narrowed (or presents a high MS), then breathing during sleep may be compromised.

The current authors postulated that the combination of nasal obstruction with a high MS could increase the risk of having obstructive disordered breathing during sleep and therefore the aim of this prospective study was to verify this hypothesis in a large group of patients referred to the authors' institution for suspicion of snoring or OSAS.

\section{Methods}

\section{Subjects}

All the patients referred to the Cliniques Universitaires St-Luc (Université Catholique de Louvain, Brussels, Belgium) for suspicion of SDB during a period of 6 months were included. A total of 220 patients were examined and had a full-night polysomnography (PSG). The exclusion criteria were abnormal lung function, severe cardiac failure responsible for Cheynes-Stokes breathing, upper airway neoplasm and acute rhinitis resulting in nasal obstruction at the time of the study. A complete physical examination was followed by an ear, nose and throat 
examination, which included anterior rhinoscopy, endonasal flexible endoscopy and both anterior and posterior semi-quantitative rhinomanometry [11]. The other routine tests were plain chest radiography, electrocardiogram and thyroid function tests. All subjects had standard spirometric measurements, maximal inspiratory and expiratory flow/volume curves, a carbon monoxide transfer test and arterial blood gas measurements.

\section{Physical examination}

One of the authors (G. Liistro) examined all the subjects during their stay in hospital, i.e. within $24 \mathrm{~h}$ of the PSG. The physical examination consisted of the measurement of the MS, neck circumference, height and weight, and the assessment of nasal patency. MS was determined as previously described [5].

The patients were asked to open the mouth wide with voluntary protrusion of the tongue without phonation. Oropharyngeal crowding was graded as follows. Grade I: tonsils, pillars and soft palate were clearly visible. Grade II: the uvula, pillars and upper pole were visible. Grade III: only part of the soft palate was visible; the tonsils, pillars and base of the uvula could not be seen. Grade IV: only the hard palate was visible.

To assess nasal patency, the patients were asked to gently block one nostril at a time with one finger, being careful to avoid the compression of the other nostril. The patient then inspired through the unoccluded nostril and the manoeuvre was repeated with the other nostril. If the examiner heard nasal obstruction, the patient was asked whether nasal occlusion was habitual or not. Unilateral and bilateral nasal obstructions were quoted equally. In a sample of 30 patients, only three were clinically estimated as having no nasal obstruction, whereas total nasal resistance measured by anterior rhinomanometry was moderately above the normal values [11] (fig. 1). MS and nasal permeability assessments took $<2$ min.

\section{Polysomnography}

A full-night diagnostic PSG was performed in each subject according to standard criteria as described previously [12]. A microphone was glued onto the anterior face of the patients' neck, upon the larynx. Airflow was monitored by three thermocouples placed in front of the mouth and each nostril and linked to independent channels. Body position was recorded (Pro-Tech body position sensor; Pro Tech, Woodinville, WA, USA) via one channel.

All signals were recorded with a digital acquisition system (OSG Brainlab, Antwerp, Belgium). Sleep and respiratory parameters were recorded at the following sampling rates: electro-oculogram (EOG) (two channels, right and left), $128 \mathrm{~Hz}$; chin electromyogram (EMG) (one channel), $512 \mathrm{~Hz}$; electroencephalogram (EEG) (three channels, C4-A1, C3-A2 and C4-O2), $128 \mathrm{~Hz}$; electrocardiogram (ECG), $128 \mathrm{~Hz}$; thoracoabdominal movements, $64 \mathrm{~Hz}$; and arterial oxygen

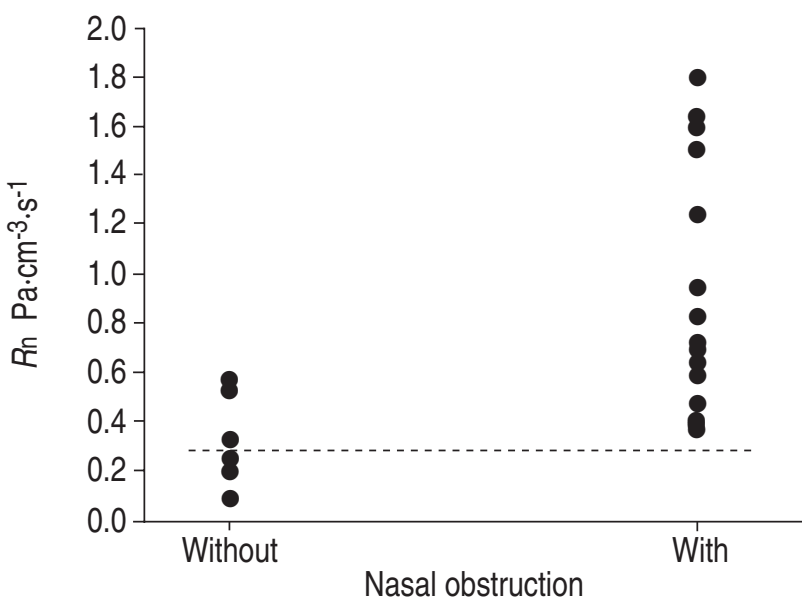

Fig. 1.-Relationship between total nasal resistance $(R \mathrm{n})$ measured by anterior rhinomanometry and clinical estimation of nasal patency in a sample of 30 subjects without and with nasal obstruction. ----: upper level of the normal values for $R$ n. Three patients quoted as "without nasal obstruction" had $R \mathrm{n}$ values above the normal range.

saturation and pulse rate, $16 \mathrm{~Hz}$, as previously described [13]. Snoring was designated on the basis of the characteristic microphone trace during sleep.

The apnoea/hypopnoea index (AHI) was the number of $>4 \%$ arterial oxygen desaturations per hour of sleep. A movement arousal (MA) was defined as the reappearance of an alpha-rhythm in the EEG during a sleep epoch, accompanied by an increase in EMG, both lasting for $\geqslant 2 \mathrm{~s}$ [14]. The MA index (MAI) is the number of MA per hour of sleep. Snoring index was defined as the number of 30-s sleep epochs with at least one snore divided by the total number of sleep epochs multiplied by 100 . The diagnosis of OSAS was retained if the subject had an AHI of $>15$ and an MAI of $>28$.

\section{Statistical analysis}

The relationship between MS and AHI was assessed by Spearman's rank correlation coefficient $\left(\mathrm{r}_{\mathrm{s}}\right)$. The AHI was compared between the Mallampati classes by Kruskal-Wallis one-way analysis of variance. The Pearson Chi-squared test was used to verify the association between MS and OSAS. Relative risk for OSAS was calculated according to standard methods. The difference in the variables compared between OSAS and non-OSAS was analysed using the standard twosample t-test. The relationship between continuous variables was analysed by standard linear regression.

\section{Results}

At the end of the recruitment, 220 subjects were examined and had full-night PSG. Eighteen subjects were excluded because of abnormal lung function tests $(n=8)$, laryngeal neoplasm $(n=1)$, severe cardiac 
dysfunction with Cheyne-Stokes periodic breathing $(n=1)$, acute rhinitis $(n=2)$ or incomplete polysomnographic data $(n=6)$. Table 1 shows the average anthropometric and polysomnographic data of the 202 subjects included in the study. Nasal obstruction was more frequent in the OSAS (32 of 10) than the non-OSAS patients (93 of 67) $(\mathrm{p}=0.03)$.

A significant correlation was found between the MS and the AHI measured during sleep $\left(r_{s}=0.264\right.$, $\mathrm{p}<0.001)$. The Pearson Chi-squared test showed a significant association $(\mathrm{p}=0.007)$ between $\mathrm{MS}$ and OSAS for the whole cohort of patients. However, there was no association between MS and OSAS in patients without nasal obstruction $(\mathrm{n}=77)$. Conversely, both the association between MS and OSAS $(\mathrm{p}=0.002)$ and the relationship between MS and AHI were more significant in the subgroup of subjects with nasal obstruction $\left(\mathrm{r}_{\mathrm{s}}=0.402, \mathrm{p}<0.001\right)$ as compared with the whole group of subjects. Figure 2 shows the difference in AHI between the four Mallampati classes in the subjects with or without nasal obstruction. A significant difference in AHI between Mallampati classes was found only in the group with nasal obstruction $(\mathrm{p}<0.001)$. The relative risk of OSAS with an MS of 3 or 4 was 1.95 (95\% confidence interval (CI) 1.10-3.48) for the whole group and $2.45(95 \%$ CI $1.23-4.84)$ in patients with nasal obstruction.

Conversely, the difference in AHI between both groups was significant only within class 4 of Mallampati. As in a previous study from the present authors' laboratory, a significant relationship was found between AHI and MAI $(r=0.750, p<0.001)$ [14].

There was a significant correlation between body mass index $(\mathrm{BMI})$ and $\mathrm{MS}\left(\mathrm{r}_{\mathrm{s}}=0.208, \mathrm{p}<0.01\right)$ and between neck circumference and MS $\left(r_{s}=0.216\right.$, $\mathrm{p}<0.01)$. However, these correlations were not significant when calculated separately in the subgroups of OSAS patients and in non-OSAS patients. Neck circumference was significantly higher in OSAS than in non-OSAS for the whole group (table 1). Within each Mallampati class, BMI was significantly higher in OSAS than in non-OSAS for the whole group but the difference was significant within Mallampati class 4 only. No correlation was found between MS and neck circumference or BMI in the subjects without nasal obstruction. Table 2 summarises the correlation coefficients obtained from the relationship between $\mathrm{AHI}$ and MS, BMI and neck circumference. Contrary to $\mathrm{MS}$, the relationships between AHI and BMI or neck circumference were also significant in the
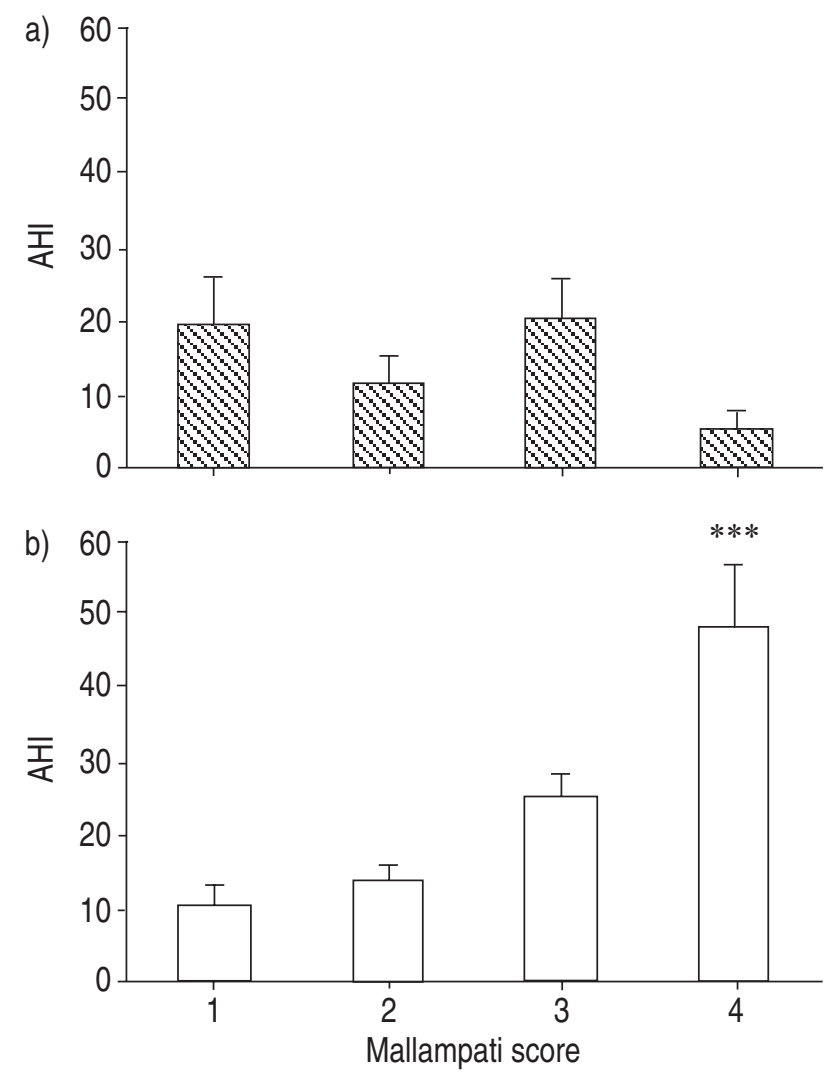

Fig. 2.-Average \pm SEM apnoea/hypopnoea index (AHI) of the patients classified according to their Mallampati score (MS), a) without or b) with nasal obstruction. A significant difference between MS was found in the subgroup with nasal obstruction $(\mathrm{p}<0.001)$. ***: difference of AHI between a) and b) within class $4, \mathrm{p}<0.001$.

subgroup of subjects without nasal obstruction. Figure 3 presents the distribution of the subjects with an MS of 3 and 4. This scatterplot of the relationship between MAI and AHI shows that the patients with an OSAS and MS of 4 all had nasal obstruction. Moreover, this subgroup of OSAS patients had a higher BMI than the other subjects in the same Mallampati class but without OSAS, whereas there was no difference in BMI within class 3 of Mallampati. Snoring index did not correlate independently with MS; it correlated with AHI $(p=0.004)$ and was not associated with MS in patients without OSAS.

Table 1. - Anthropometric data of the subjects

\begin{tabular}{lcccccc}
\hline & Subjects $\mathrm{n}$ & Age yrs & BMI kg.m ${ }^{-2}$ & Sex M:F & Neck circ. cm & AHI \\
\hline Whole group & 202 & $49.4 \pm 11.7$ & $29.5 \pm 5.9$ & $161: 41$ & $41.3 \pm 3.9$ & $19.1 \pm 22.4$ \\
Non-OSAS & 160 & $48.5 \pm 11.9$ & $28.6 \pm 5.0$ & $122: 38$ & $40.6 \pm 3.8$ & $12.0 \pm 16.5$ \\
OSAS & 42 & $53.0 \pm 10.4$ & $33.2 \pm 7.3$ & $39: 3$ & $43.7 \pm 3.3$ & $46.2 \pm 21.6$ \\
p-value & $<0.001$ & 0.02 & $<0.001$ & 0.02 & $<0.001$ & $<0.001$ \\
\hline
\end{tabular}

BMI: body mass index; M: males; F: females; Neck cir.: neck circumference; AHI: apnoea/hypopnoea index; OSAS: obstructive sleep apnoea index. The p-values were obtained by the Chi-squared test for number of the subjects, Fisher's exact test for the sex ratio and by unpaired t-test for age, BMI, neck circ. and AHI. 
Table 2. - Relationship between apnoea/hypopnoea index ( $\mathrm{AHI}$ ) and Mallampati score, body mass index (BMI) and neck circumference (Neck circ.)

\begin{tabular}{|c|c|c|c|c|c|c|c|c|c|}
\hline & \multicolumn{9}{|c|}{ AHI } \\
\hline & \multicolumn{3}{|c|}{ Whole group } & \multicolumn{3}{|c|}{ Without nasal obstruction } & \multicolumn{3}{|c|}{ With nasal obstruction } \\
\hline & $\mathrm{r}$ & $95 \% \mathrm{CI}$ & $\mathrm{p}$-value & $\mathrm{r}$ & $95 \% \mathrm{CI}$ & p-value & $\mathrm{r}$ & $95 \% \mathrm{CI}$ & p-value \\
\hline $\mathrm{MS}^{\#}$ & 0.264 & $0.131-0.388$ & $<0.001$ & 0.023 & $-0.202-0.246$ & $>0.05$ & 0.402 & $0.244-0.540$ & $<0.001$ \\
\hline BMI & 0.633 & $0.542-0.709$ & $<0.001$ & 0.678 & $0.535-0.783$ & $<0.001$ & 0.613 & $0.490-0.712$ & $<0.001$ \\
\hline Neck circ. & 0.551 & $0.447-0.640$ & $<0.001$ & 0.519 & $0.334-0.666$ & $<0.001$ & 0.583 & $0.454-0.688$ & $<0.001$ \\
\hline
\end{tabular}

95\% CI: $95 \%$ confidence intervals for r. ${ }^{*}$ : the Spearman correlation.
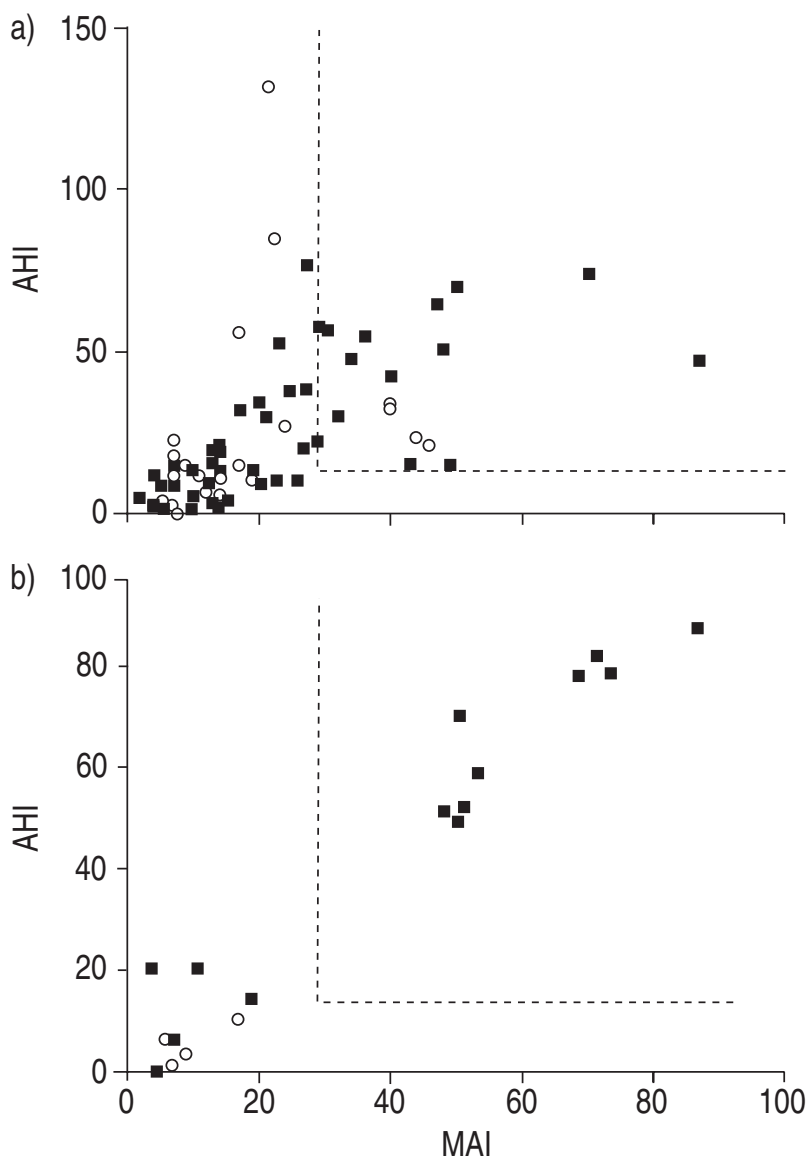

Fig. 3.-Relationship between movement arousal index (MAI) and apnoea/hypopnoea index (AHI) in the patients with a Mallampati score (MS) of a) 3 and b) 4. $\mathbf{a}$ : subjects with nasal obstruction; $\bigcirc$ : without nasal obstruction; ----: separates obstructive sleep apnoea syndrome (OSAS; above line) from non-OSAS (below line) patients. Average \pm SD body mass index (BMI) for the two groups were as follows. MS 3 OSAS: $32.6 \pm 6.3 \mathrm{~kg} \cdot \mathrm{m}^{-2}$; MS 3 nonOSAS: $29.9 \pm 5.6 \mathrm{~kg} \cdot \mathrm{m}^{-2}$; MS 4 OSAS: $40.4 \pm 8.8 \mathrm{~kg} \cdot \mathrm{m}^{-2}$; MS 4 non-OSAS: $27.7 \pm 4.4 \mathrm{~kg} \cdot \mathrm{m}^{-2}$. Note that the OSAS patients all had nasal obstruction with a higher BMI $(\mathrm{p}=0.011)$.

\section{Discussion}

It has been shown here that the presence of a high MS with nasal occlusion was associated with an increased risk of OSAS in a group of 202 subjects referred for assessment of SDB.
Patients with abnormal lung or cardiac function were excluded to avoid confounding factors. Patients with upper airway neoplasm were also excluded because this cause of upper airway obstruction is not detectable with the simplified examination used in this study.

The role of nasal obstruction in SDB has been largely investigated in the last 2 decades. Experimentally induced nasal obstruction increases the number of obstructive events during sleep $[9,10]$. The presence of unilateral or bilateral nasal obstruction was associated either with snoring or OSAS in a large series of patients [15]. However, these authors found no direct relationship between nasal resistance measured during wakefulness and SDB. More recently, LofAso et al. [7] have shown that, in a large series $(n=541)$ of patients referred to a sleep clinic for snoring and daytime somnolence, nasal resistance was an independent risk factor for OSAS. These studies report different results, although they used different statistical analysis and methods for measuring nasal resistance. However, in the study of LOFASO et al. [7], the contribution of nasal resistance to the OSAS was weak: $2.3 \%$ of the variance of $\mathrm{AHI}$.

The method used in this study for assessing nasal permeability is, of course, rough and approximate. Nevertheless, it rapidly indicates whether a nasal dyspermeability exists or not. In a sample of 30 patients, three were quoted clinically as "nonobstructed", whereas rhinomanometry showed that the nasal resistance was moderately elevated. In these subjects, the observer did not perceive a bilateral and symmetrical increase in nasal resistance. Several factors influence nasal permeability, which is variable in time. Sleep does not significantly influence total nasal resistance $[16,17]$. This is true only in the absence of marked mucosal anomaly or structural deviation of the nasal septum [18].

The contribution of both high MS and nasal obstruction have not been studied before and the usefulness of a modified MS (MMS) in SDB has only been investigated in one study [4]. In this study, the patients opened their mouths without protruding the tongue, at variance with the standard MS used in the current study protocol. This study reported a significant correlation $(\mathrm{r}=0.340, \mathrm{p}<0.001)$ between the MMS and the respiratory disturbance index (RDI; the sum of apnoeas and hypopnoeas per hour of sleep) in a group of 172 patients selected on the basis of a sleep 
questionnaire. These authors used a standard linear regression analysis to study the relationship between the MMS and RDI. The present authors used the rank correlation coefficient because the Mallampati classes are ordered categorical variables [19]. However, if the Pearson's correlation coefficient is computed with the data from the current study, comparable values are obtained. The correlation between MS and AHI was $0.293, \mathrm{p}<0.001$ for the whole group and $\mathrm{r}=0.465$, $\mathrm{p}<0.001$ for the subgroup with nasal obstruction.

The clinical examination was performed on awake subjects but could it change during sleep? Тнам et al. [20] observed that posture had a very small effect on the MS in a group of 128 subjects. Supine position displaced the distribution of the MS slightly towards the higher classes of Mallampati. The current authors have found a significant correlation between BMI and neck circumference with AHI. This is consistent with the literature [2]. Contrary to BMI or neck circumference, MS was not correlated with AHI in the subjects without nasal obstruction. This shows that BMI and collar size are independent predictors of AHI whereas MS is associated with AHI only when nasal obstruction is present. This confirms the authors' basic hypothesis that the association of impaired nasal and oral airways is a risk factor for OSAS. This association was consistent with a higher risk of SDB but did not permit the differentiation between subjects with or without OSAS. In this study, a clinical association, but not a diagnostic tool, has been found. However, this simple assessment of nasal and oral patency may be added to the routine physical examination of the patients with sleep complaints. This study was conducted in a group of patients suspected of having either snoring every night or OSAS. No conclusions can be drawn for a nonselected population, but the presence of a high MS and nasal obstruction is easily detectable by the anaesthetist in patients who must undergo a general anaesthesia. A previous report from the current authors' group has shown that patients with OSAS have a greater risk of postoperative complications [21].

In conclusion, this study shows that simple and rapid elements such as the determination of Mallampati score and the estimation of nasal permeability are useful for the clinical examination of the patients suspected of obstructive sleep apnoea syndrome. Conversely, the association of a high Mallampati score and nasal obstruction must attract the attention of the anaesthetist and the sleep physician.

\section{References}

1. Davies RJ, Ali NJ, Stradling JR. Neck circumference and other clinical features in the diagnosis of the obstructive sleep apnoea syndrome. Thorax 1992; 47: 101-105.

2. Stradling JR, Crosby JH. Predictors and prevalence of obstructive sleep apnoea and snoring in 1001 middle aged men. Thorax 1991; 46: 85-90.

3. Kushida CA, Efron B, Guilleminault C. A predictive morphometric model for the obstructive sleep apnea syndrome. Ann Intern Med 1997; 127: 581-587.
4. Friedman M, Tanyeri H, La Rosa M, et al. Clinical predictors of obstructive sleep apnea. Laryngoscope 1999; 109: 1901-1907.

5. Mallampati SR, Gatt SP, Gugino LD, et al. A clinical sign to predict difficult tracheal intubation: a prospective study. Can Anaesth Soc J 1985; 32: 429-434.

6. Atkins M, Taskar V, Clayton N, Stone P, Woodcock A. Nasal resistance in obstructive sleep apnea. Chest 1994; 105: 1133-1135.

7. Lofaso F, Coste A, d'Ortho MP, et al. Nasal obstruction as a risk factor for sleep apnoea syndrome. Eur Respir J 2000; 16: 639-643.

8. Young T, Finn L, Kim H. Nasal obstruction as a risk factor for sleep-disordered breathing. The University of Wisconsin Sleep and Respiratory Research Group. J Allergy Clin Immunol 1997; 99: S757-S762.

9. Zwillich CW, Pickett C, Hanson FN, Weil JV. Disturbed sleep and prolonged apnea during nasal obstruction in normal men. Am Rev Respir Dis 1981; 124: 158-160.

10. Suratt PM, Turner BL, Wilhoit SC. Effect of intranasal obstruction on breathing during sleep. Chest 1986; 90: 324-329.

11. Cole P, Roithmann R, Roth Y, Chapnik JS. Measurement of airway patency. A manual for users of the Toronto systems and others interested in nasal patency measurement. Ann Otol Rhinol Laryngol Suppl 1997; 171: 1-23.

12. Atlas task force of the American Sleep Disorders Association. EEG Arousals: scoring rules and examples. Sleep 1992; 15: 174-184.

13. Parreira VF, Delguste P, Jounieaux V, Aubert G, Dury M, Rodenstein DO. Glottic aperture and effective minute ventilation during nasal two-level positive pressure ventilation in spontaneous mode. Am J Respir Crit Care Med 1996; 154: 1857-1863.

14. Collard P, Dury M, Delguste P, Aubert G, Rodenstein DO. Movement arousals and sleep-related disordered breathing in adults. Am J Respir Crit Care Med 1996; 154: 454-459.

15. Miljeteig $\mathrm{H}$, Hoffstein V, Cole $\mathrm{P}$. The effect of unilateral and bilateral nasal obstruction on snoring and sleep apnea. Laryngoscope 1992; 102: 1150-1152.

16. Wheatley JR, Tangel DJ, Mezzanotte WS, White DP. Influence of sleep on alae nasi EMG and nasal resistance in normal men. J Appl Physiol 1993; 75: 626-632.

17. Hudgel DW, Robertson DW. Nasal resistance during wakefulness and sleep in normal man. Acta Otolaryngol Stockh 1984; 98: 130-135.

18. Miljeteig H, Cole P, Haight JS. Nasal resistance in recumbency and sleep. Rhinology 1995; 33: 82-83.

19. Altman DG, Gore SM, Gardner MJ, Pocock SJ. Statistical guidelines for contributors to medical journals. In: Gardner MJ, Altman DG, eds. Statistics with confidence-confidence intervals and statistical guidelines. London, British Medical Journal, 1989; pp. 83-100.

20. Tham EJ, Gildersleve CD, Sanders LD, Mapleson WW, Vaughan RS. Effects of posture, phonation and observer on Mallampati classification. $\mathrm{Br} J$ Anaesth 1992; 68: 32-38.

21. Rennotte MT, Baele P, Aubert G, Rodenstein DO. Nasal continuous positive airway pressure in the perioperative management of patients with obstructive sleep apnea submitted to surgery. Chest 1995; 107: $367-374$. 